\title{
Síndrome de Boca Ardiente: actualización
}

\author{
Perdomo Lovera M, Chimenos Küstner E
}

\section{RESUMEN}

El síndrome de la boca ardiente (SBA) es una entidad nosológica, más frecuente en mujeres menopáusicas. Su principal característica es una sintomatología urente y/o dolorosa de la mucosa bucal (principalmente en la lengua), sin hallazgos clínicos aparentes que expliquen la aparición de esta sensación. Se encuentra relacionado con una serie de factores locales y sistémicos posibles desencadenantes, por lo que se considera de etiología multifactorial. El tratamiento va dirigido a mejorar la sintomatología y controlar los factores. Las terapias propuestas consistentes en control de factores locales, factores sistémicos y psicológicos y algunas indicaciones farmacológicas, proporcionan resultados mediocres. En términos generales, el SBA continúa siendo objeto de estudios en el ámbito de la medicina bucal en busca de un diagnóstico más preciso y de una terapéutica más eficaz.

Palabras clave: Síndrome de boca ardiente, Glosodinia, Menopausia.

\begin{abstract}
Burning mouth syndrome (BMS) is a nosological entity, frequent in women in the menopausal period, which principal characteristic is a burning and/or pain sensation of the oral cavity (principally in the tongue). However, the oral mucosa often appears normal, with no apparent organic cause to account for the symptomatology. This syndrome is found to be related which a series of local and systemic factors as possible cause. Therefore is to be considerate a pathology with multifactorial etiology. The treatment is directed to improve the symptomatology and to control the factors. The proposal therapies, related to the control of local, systemic and psychological factors and some pharmacological indications, provide poor results. In general terms the BMS is still the object of many studies in the environment of the oral medicine, in order to search several aspects of diagnose and a more effective therapy.
\end{abstract}

Key words: Burning mouth syndrome, glossodynia, menopause.

* $\quad$ Facultad de Odontología, Universidad de Barcelona.

Perdomo Lovera M, Chimenos Küstner E. Síndrome de Boca Ardiente: actualización. Av. Odontoestomatol 2003; 19-4: 193-202

\section{INTRODUCCIÓN}

El síndrome de la boca ardiente (SBA) es un cuadro clínico muy frecuente, caracterizado por una sensación espontánea anormal descrita por el paciente como quemazón, ardor, escozor, que afecta la mucosa bucal, generalmente en ausencia de datos clínicos y de laboratorio que justifiquen estos síntomas (1-7). La zona de mayor afectación es la lengua (punta y bordes), por lo que se le ha llamado "glosodinia" y "glosopirosis" (lengua ardiente) (7-9). Comúnmente, además de ardor, los pacientes experimentan síntomas tales como alteración del gusto, sensación punzante, sequedad, intolerancia a las prótesis (7). Estas condiciones se presentan como parte del SBA o bien de forma alternativa (2). Es más frecuente en mujeres en edades medias-altas de la vida. Concretamente es típico en mujeres perimenopáusicas (10).

La falta de signos clínicos aumenta su complejidad y hace difícil su diagnóstico, al cual se le ha prestado atención esporádica en la bibliografía, durante los 
últimos 50 años. Sigue siendo una materia objeto de considerable controversia y falta de comprensión. En la actualidad, diversos investigadores pretenden dar respuesta a un sinfín de interrogantes con respecto a ese tema. La multiplicidad de factores que se relacionan con esta entidad nosológica y que, de una u otra forma, intervienen en la aparición de la sintomatología que lo caracteriza, ha hecho que actualmente este tema sea uno de los más estudiados en el área de la medicina bucal.

\section{EPIDEMIOLOGÍA}

Entre la población general se puede decir que se presenta con una frecuencia que oscila desde un 0,7 \% a un $4,5 \%(6,10,11)$. La incidencia de SBA en adultos varía en cada estudio realizado, por lo que no se ha establecido una prevalencia fidedigna para la población general. Existen más probabilidades de que ocurra en mujeres que en hombres, en una relación aproximada de 3:1 a 9:1 (6, 7, 10); las mujeres más frecuentemente afectadas se encuentran en el período menopáusico o postmenopáusico, hasta un $30 \%$ de prevalencia (3-7, 11-17). Aparece con mayor frecuencia en edades medias-altas de la vida. La edad promedio en la que aparece es entre 50-60 años (rango: 36-86 años) (2). Descubrir este síndrome en pacientes con menos de 30 años es raro, no habiendo sido nunca descrito en niños, ni adolescentes $(4,7)$. Según Okeson (8), la edad de aparición en mujeres es entre los 40 y 49 años y en los hombres es entre los 30 y 59 años. El hecho de que su diagnóstico sea dificultoso hace más difícil determinar sus índices epidemiológicos (12).

\section{CLÍNICA}

Ardor, escozor, hormigueo, adormecimiento, quemazón, son algunos de los calificativos utilizados por los pacientes para definir el disconfort que les causa lo que en clínica se conoce como síndrome de boca ardiente. Se define como una sensación de ardor en cualquier parte de la mucosa bucal, en ausencia de hallazgos clínicos y de laboratorio (6). La sintomatología ha sido descrita como una molestia continua, con periodos de agudización (crisis), pero en general de tipo crónico, de inicio espontáneo, sin factor iden- tificable de precipitación, a no ser el estrés y otros factores psicológicos. Aproximadamente 1/3 de los pacientes relaciona la aparición de los síntomas con un tratamiento odontológico, una enfermedad reciente o una medicación no habitual (incluyendo antibióticos) (6). Sin importar la naturaleza de la molestia, una vez que ésta aparece puede persistir muchos años.

El ardor bucal suele aumentar progresivamente durante el día, alcanzando su intensidad más alta al final de la tarde. En la mayoría de pacientes el dolor está ausente por la noche; normalmente no los despierta, pero les dificulta conciliar el sueño $(6,7)$. Quizás debido a esta dificultad, al dolor constante, o a ambos, estos pacientes presentan a menudo cambios de humor, incluyendo irritabilidad, ansiedad y depresión (14).

Existen autores que categorizan el SBA basándose en la gravedad de sus síntomas como: suave, moderado o severo. Afirman que el moderado es el que ocurre con más frecuencia y que es comparable con el dolor dental $(1,15,18)$. Se han definido 3 tipos de SBA (Tabla 1) basándose en las fluctuaciones diurnas de los síntomas (clasificación realizada por Lamey y Lewis en 1989 ) (19).

El sitio de afectación más frecuente es la lengua $(67,9 \%)$, siendo más específicamente la punta y los bordes de ésta los más molestos. En segundo lugar de frecuencia de afectación, para algunos autores, se encuentra en la porción mucosa del labio inferior y el paladar duro $(6,7,13,15)$. Por el contrario, según Bergdahl y Bergdahl (17), la segunda zona de la cavidad bucal más afectada por la molestia es la encía, en un 11,3\%. Algunos pacientes no saben definir qué zona es la que le molesta más; por esto suelen expresar que sienten ardor en "toda la boca" $(7,17)$. En un estudio realizado por Chimenos y cols. (3), de un total 44 pacientes, 32 refirieron solo afectación lingual y los 12 restantes afectación añadida de otras zonas de la cavidad bucal.

Se encuentra poca información sobre el curso natural del síndrome de boca ardiente. Se ha publicado que puede existir una recuperación parcial espontánea en 6 a 7 años después del inicio. Esta puede estar precedida por cambios de sintomatología y episodios libres 


\begin{tabular}{|c|c|c|c|}
\hline \multicolumn{4}{|c|}{ TABLA 1.- CLASIFICACIÓN CLÍNICA DEL SBA Lamey y Lewis (19), modificada } \\
\hline TIPO & Síntomas & Porcentaje de & Relación con agente causal \\
\hline I & $\begin{array}{l}\text { No existen al levantarse } \\
\text { Aumenta por la tarde }\end{array}$ & $65 \%$ & $\begin{array}{l}\text { Déficits nutricionales } \\
\text { Trastornos psicológicos }\end{array}$ \\
\hline II & $\begin{array}{l}\text { Continuos } \\
\text { Todo el día }\end{array}$ & $26 \%$ & Ansiedad crónica \\
\hline III & $\begin{array}{l}\text { Intermitentes } \\
\text { Días libres de síntomas }\end{array}$ & $10 \%$ & Alergia alimentaria \\
\hline
\end{tabular}

de sensación dolorosa; no se ha observado ningún factor clínico que preceda las recuperaciones $(6,15)$.

La mayoría de los estudios han encontrado que el ardor bucal está acompañado con frecuencia por otros síntomas, incluyendo boca seca y alteraciones del gusto (disgeusias), sabor metálico, sabor amargo o combinaciones de varios de ellos y/o cambio en intensidad de la percepción del gusto $(6,20-22)$. Además pueden aparecer dificultades para tragar, dolor facial o dental atípicos (23).

\section{ETIOPATOGENIA}

Muchos investigadores han intentado, a lo largo de los años, explicarse la causa del síndrome de la boca ardiente. De estos intentos científicos han brotado muchas ideas que han sido refutadas por otros, con lo cual en la actualidad la duda persiste. La mayoría de los pacientes con boca ardiente tienen asociados factores que pueden considerarse favorecedores de un trastorno de la percepción de la mucosa bucal (24). El cuadro clínico del SBA es complejo, lo que ha creado una dificultad importante para entenderlo. ¿Qué lo produce? Esta pregunta todavía en la actualidad no tiene una respuesta concreta; se plantean numerosos factores relacionados. Sin embargo, cada uno de estos explica la aparición del síntoma solamente en pequeños grupos de pacientes. Cualquiera de ellos puede estar reflejado en la historia de un individuo con SBA y no necesariamente aparecer en otro con el mismo diagnóstico.

Se atribuye a esta entidad nosológica un carácter etiológico multifactorial. Un hecho importante a señalar es que, en muchas ocasiones, corregir o eliminar estos agentes presuntamente causales no determinan la mejoría o desaparición de la sintomatología inicial. Los diferentes factores relacionados con la etiopatogenia de este síndrome se han dividido en: locales, sistémicos y psicológicos.

\section{FACTORES LOCALES}

Se consideran en este apartado factores capaces de desencadenar la sintomatología urente afectando la mucosa bucal directamente; generalmente actúan por irritación localizada de la misma y pueden ser de distinta naturaleza: físicos (mecánicos, térmicos, eléctricos), químicos (alcohol: contenido en bebidas o colutorios) $(3,7)$ o biológicos, entre los cuales se pueden incluir algunas bacterias y hongos $(1,7,16$, 21-30). Los hábitos parafuncionales $(7,16,31)$ y la xerostomía (subjetiva u objetiva) se encuentran íntimamente ligados a este síndrome $(12,15-17,32$ 39). Sustancias de uso diario, como por ejemplo los dentífricos y otros cosméticos, pueden producir reacciones que desencadenen el síndrome (16, 4044). También se asocian factores ligados a la actividad neural sensitiva de la mucosa bucal, pudiéndose encontrar disminución en el umbral del dolor y alteraciones del gusto tales como la "fantogeusia" (sabor a algo sin que halla nada dentro de la boca) (22, 45-47) (Tabla 2).

\section{FACTORES SISTÉMICOS}

Gran variedad de entidades nosológicas sistémicas han sido consideradas factores relacionados con la 


\begin{tabular}{|c|c|}
\hline FACTORES LOCALES & RELACIÓN CON SBA \\
\hline $\begin{array}{l}\text { Tratamientos odontológicos recientes } \\
\text { Hábitos tóxicos }\end{array}$ & $\begin{array}{l}\text { Pueden desencadenar los síntomas ( } 64 \% \text { de casos) } \\
\text { - Alcohol (acción química) } \\
\text { - Tabaco (acción térmica) }\end{array}$ \\
\hline Agentes infecciosos & $\begin{array}{l}\text { - Candidiasis: Cándida albicans, Candida glabrata } \\
\text { - Espiroquetas } \\
\text { - Helicobacter pylori } \\
\text { - Infección periodontal }\end{array}$ \\
\hline Glositis migratoria benigna o lengua geográfica & $\begin{array}{l}\text { Existe una asociación entre esta entidad clínica y el } \\
\text { SBA ( } 15 \% \text { de casos) }\end{array}$ \\
\hline Traumatismo & $\begin{array}{l}\text { Roce producido por prótesis mal ajustadas y de aca- } \\
\text { bado deficiente ( } 50 \% \text { de casos) }\end{array}$ \\
\hline Hábitos parafuncionales & $\begin{array}{l}\text { - Empuje lingual } \\
\text { - Frotamiento continuo de la mucosa bucal sobre } \\
\text { prótesis y dientes alterados } \\
\text { - Succión de lengua, labios y carrillos } \\
\text { - Bruxismo }\end{array}$ \\
\hline Cantidad y calidad de la saliva & $\begin{array}{l}\text { - } 3 / 4 \text { partes de los pacientes presentan sensación de } \\
\text { boca seca } \\
\text { - Volumen del flujo salival (estimulado y no estimula- } \\
\text { do) NORMAL } \\
\text { - Disminución cuantificada del flujo salival en pacien- } \\
\text { tes de edad avanzada, polimedicados } \\
\text { - Hallazgos de irregularidades en las concentraciones } \\
\text { de componentes de la saliva }\end{array}$ \\
\hline Reacciones alérgicas & $\begin{array}{l}\text { - Materiales dentales } \\
\text { - Sustancias alimentarias } \\
\text { - Cosméticos } \\
\text { - Dentífricos }\end{array}$ \\
\hline Mecanismos neuronales & $\begin{array}{l}\text { - Condición neuropatológica del sistema nervioso } \\
\text { central y/o periférico } \\
\text { - Disfunciones de vías periféricas del dolor } \\
\text { - Alteraciones del umbral del dolor } \\
\text { - Alteración en la conducción nerviosa }\end{array}$ \\
\hline Función del gusto & $\begin{array}{l}\text { - Entre los pacientes, supergustativos hay un alto índi- } \\
\text { ce de SBA } \\
\text { - Fantogeusia aparece como síntoma anexo } \\
\text { - Posible daño en nervios gustativos } \\
\text { - Dificultad para diferenciar sabores (ácido y salado) }\end{array}$ \\
\hline
\end{tabular}


aparición del SBA. Estados carenciales como deficiencias vitamínicas, entre las que destacan los niveles bajos de vitamina B12 $(48,49)$ y otros como vitamina $\mathrm{B} 6$, ácido fólico y vitamina $\mathrm{C}$, pueden desencadenar ardor bucal como sintomatología concomitante. Así, algunos de los pacientes diagnosticados de anemia refieren síntomas como la glosodinia o glosopirosis.

Con respecto al sistema endocrino, hay dos trastornos que se relacionan con el SBA. Uno de ellos es la disminución hemática de estrógenos, que repercute sobre la mucosa bucal provocando ardor, alteraciones del gusto y xerostomía $(10,16,24)$. Otra afectación endocrina relacionada con el SBA es la diabetes mellitus no controlada, en cuyo caso los síntomas desaparecen al normalizar la glicemia del paciente $(24,50)$.

Numerosos factores sistémicos se han relacionado con el SBA; muchos de ellos son entidades nosológicas carenciales, como deficiencias vitamínicas y anemias, así como alteraciones hormonales e inmunológicas.

\section{Fármacos}

Existe una gran diversidad de fármacos que, entre sus efectos, producen xerostomía, lo que está íntimamente relacionado con el ardor bucal. Entre ellos se encuentran antihistamínicos, neurolépticos, algunos antihipertensivos, antiarrítmicos y benzodiacepinas, principalmente (24). Para algunos autores la medicación con L-tiroxina es el factor farmacológico más importante relacionado con el SBA (19). Los resultados de Bergdahl y cols. (19) confirman una relación entre los medicamentos y el SBA, que se agrava con la interacción de más factores, como enfermedades sistémicas y condición mental de los pacientes.

\section{FACTORES PSICOLÓGICOS}

Generalmente se ha asociado el SBA a un estado de ansiedad importante, que el paciente muestra al describir su proceso clínico, desde el primer momento de la exploración. No se ha podido explicar con claridad la fisiopatología de esta relación, por lo cual no se sabe si aparece el ardor bucal a causa del trastor- no psicológico, o éste es una consecuencia de la sintomatología. Los factores psicológicos y sociológicos desempeñan un importante papel en los desórdenes dolorosos faciales y orofaciales $(16,19$, 24, 26, 51, 52). El empleo de diversas pruebas y escalas de valores ha demostrado una presencia frecuente de cambios psicológicos en estos pacientes. Los cambios más comunes son: depresión, ansiedad, inadaptabilidad social e inestabilidad emocional (52). La personalidad de los pacientes con SBA es similar a la de otros individuos que padecen enfermedades con dolor crónico. Tienden a ser depresivos, sentirse molestos, son aprensivos e introvertidos, lo cual puede ser el resultado directo de la experiencia dolorosa (16). Bergdahl y cols. (53) observaron en sus pacientes un menor nivel de socialización, mayores niveles de ansiedad somática, tensión muscular, mayor tendencia a preocuparse por su salud y más pensamientos de tristeza cuando se comparaban con pacientes control, encontrándose importantes diferencias en cuanto a personalidad y funcionamiento psicológico. Esto podría sugerir una base psicosomática del SBA (26). Con frecuencia en la anamnesis detallada aparece un hecho emocional, conflictivo, personal, laboral o el reconocimiento de temor al cáncer. Algunos autores han identificado a los pacientes con SBA como hipocondríacos (16). Los pacientes que padecen el síndrome suelen presentar tensión psíquica y ansiedad y el ardor bucal puede ser una somatización de sus conflictos internos (30).

\section{TRATAMIENTO}

Es fundamental realizar una historia clínica completa y exhaustiva, para diagnosticar y tratar a cualquier paciente con SBA $(6,16,21)$. Debido a la característica multifactorial de su etiología, no existe un tratamiento establecido para esta entidad clínica, por lo que empíricamente se dan indicaciones para mejorar la sintomatología. La identificación del principal factor (o factores) etiológico, local, sistémico o psicógeno puede guiar al terapeuta a un diagnóstico y tratamiento (16). Se debe eliminar todo factor local o sistémico que afecte la mucosa, como por ejemplo alergias de contacto; deben controlarse los hábitos parafuncionales; deben instaurarse terapias sustitutivas (en caso de deficiencias vitamínicas y minerales). 


\section{TABLA 3.- TRATAMIENTOS DE FACTORES QUE AFECTAN LA MUCOSA BUCAL}

\author{
Enfermedades mucosas \\ Menopausia \\ Deficiencias nutricionales \\ Boca seca \\ Efectos adversos de medicamentos
}

Hacer el diagnóstico pertinente e indicar tratamiento específico

Reemplazo hormonal (s está indicado)

Suplementos orales

Hidratación, análogos salivales

Cambio de medicación (sí es posible)

\begin{tabular}{|ll|}
\hline \multicolumn{1}{|c|}{ TABLA 4.- TRATAMIENTOS UTILIZADOS EN EL SBA } \\
\hline Relacionados con factores locales & $\begin{array}{l}30 \mathrm{ml} \text { de nistatina }(100,000 \mathrm{~m} / \mathrm{ml}), 50 \mathrm{ml} \text { hidrocortisona }(10 \mathrm{mg} / \\
5 \mathrm{ml}), 60 \mathrm{ml} \text { tetraciclina }(125 \mathrm{mg} / 5 \mathrm{ml}), 120 \mathrm{ml} \text { Benadryl elixir } \\
(12.5 \mathrm{mg} / 5 \mathrm{ml}) \text {, enjuagar } 4 \text { veces al dia y escupir }\end{array}$ \\
$\begin{array}{ll}\text { Relacionados con irritabilidad nerviosa } \\
\text { Antidepresivos tricíclicos, Benzodiacepinas (solo en dosis bajas), } \\
\text { Anticonvulsivantes, Capsaicina }\end{array}$ \\
\hline
\end{tabular}

Muchos de estos pacientes toman fármacos potencialmente xerostomizantes (principalmente antihipertensivos, ansiolíticos y antidepresivos) y otros, como se muestran en la tabla 3. Si una vez practicados los tratamientos sugeridos para las condiciones locales o sistémicas persiste el ardor bucal, se puede considerar el diagnóstico de SBA (6). El tratamiento de este síndrome usualmente está orientado hacia la sintomatología y es similar al tratamiento clínico de otras neuropatías dolorosas $(6,16,54-56)$ (Tabla 4).

Los antidepresivos tricíclicos en bajas dosis actúan sobre este tipo de dolor crónico como analgésicos (57). La capsaicina tópica se usa como agente desensibilizante en pacientes con SBA, pero puede no ser aceptada por el paciente, ya que su sabor es desagradable (6). Rodrigo y col. (21) afirman que, desde su punto de vista, es necesario valorar el componente ansioso o depresivo u otras alteraciones psíquicas por las que atraviesa el paciente en el momento que llega a la consulta; aplicar una psicoterapia sencilla en la consulta supone el $80 \%$ del tratamiento, sobre todo en los pacientes relativamente estables. Es importante la interconsulta con un psicólogo o un psiquiatra, dependiendo esta elección del estado psíquico del paciente. Una vez hecho todo lo anterior, se recomienda reevaluar al paciente pasado el mes, a los 3 y 6 meses, para comprobar la eficacia del tratamiento indicado (56).

\section{DISCUSIÓN}

Tradicionalmente la boca ardiente es descrita como un síndrome de mujeres después de la menopausia, emocionalmente desequilibradas, acompañado con frecuencia por múltiples quejas somáticas. El paciente puede describir la sensación como intolerable, pero raras veces lo incapacita. Típicamente, el síntoma aumenta de gravedad a medida que avanza el día, pero no quita el sueño. Observaciones recientes indican que el síntoma lo mismo ocurre en mujeres que en hombres y no necesariamente está confinado a personas de edad avanzada. A pesar del listado, extenso, presentado de posibles factores etiopatogénicos y de apuntarse algunos mecanismos fisiopatológicos en la estructuración del síndrome, en general se sabe poco de la patogenia del mismo, así como de los factores etiológicos que pueden participar en la misma (30). En la literatura revisada parece haber una predilección basada en la experiencia clínica de que existe una combinación importante de algunos factores como enfermedad, medicación y desequilibrio emocional, que en muchos casos parecen aclarar la complejidad del síndrome. Los numerosos factores locales y sistémicos, que pueden estar relacionados con el SBA, se sabe que son capaces de producir ardor bucal, el cual puede desaparecer al controlar dichos factores. Por esta razón existen autores como Sardella y cols. (57) que se plantean qué signi- 
fica la S de las iniciales SBA: ċserá S: por síndrome, o S: por síntomas? Estos autores afirman que muchos investigadores publican sobre este tema, del cual no existe todavía una definición aceptada de forma generalizada. La frecuente asociación de otros síntomas y la complejidad que envuelve la clínica del paciente con boca ardiente hace que algunos autores prefieran usar la expresión "síndrome de boca ardiente" (58). Ellos opinan que es prudente introducir el concepto de SBA idiopático (esencial), para indicar la sensación de ardor en la cavidad oral cuando la etiología es desconocida y no se correlaciona con factores locales ni sistémicos (57). Cuando los factores locales son eliminados y los sistémicos tratados y el ardor bucal no desaparece, se recurre a la farmacoterapia crónica, con resultados muchas veces poco favorables. El hecho de que este síndrome aparezca en periodos perimenopáusicos y en la vejez, no hace sino avivar la polémica sobre si es más importante en su génesis la base orgánica (la deprivación hormonal y el natural menoscabo físico propio de edades avanzadas) o el componente emocional, también característico de estas edades, en las que se viven sensaciones de deterioro físico y abandono. Por ello ambos aspectos deben ser estudiados de una manera sumamente cuidadosa para establecer las bases de una terapéutica efectiva. El descubrimiento de los mecanismos patogénicos de este síndrome constituye en la actualidad un gran desafío, en el ámbito de la Medicina Bucal.

\section{BIBLIOGRAFÍA}

1. Huang W, Rothe MJ, Grant-Kels JM. The burning mouth syndrome. J Am Acad Dermatol 1996; 34: 91-8.

2. Zakrzewska JM. The burning mouth syndrome remains an enigma. Pain. 1995; 6: 253-7.

3. Chimenos E, Rodríguez de Rivera ME, López J. Síndrome de boca ardiente. Fisiopatología de las disgeusias. En: Ceballos A, Bullón P, Gándara JM, Chimenos E, Blanco A, Martínez A, García A (eds.). Medicina bucal práctica. Santiago de Compostela. DANÚ S.A. 2000. 565-75.

4. Carlson CR, Miller CS, Reid KI. Psychosocial pro- files of patients with burning mouth syndrome. J Orofac Pain 2000; 14: 59-64.

5. Regezi J, Sciubba J. Patología bucal. México. McGraw-Hill Interamericana Editores S.A. 1999. 142-4.

6. Grushka M, Epstein JB, Gorsky M. Burning mouth syndrome. Am Fam Physician 2002; 15: 615-20.

7. Silvestre Donat FJ, Serrano Martínez C. El síndrome de boca ardiente: revisión de conceptos y puesta al día. Medicina Oral 1997; 2: 30-8.

8. Okeson JP. Orofacial Pain. Guidelines for assessment, diagnostic and management. Illinois. Quintessence Co, Inc. 1996. 106-08.

9. Feinmman $\mathrm{CH}$, Ibbeston R. Idiopathic orofacial pain: a multidisciplinary problem. En: Feinmann C. The mouth, the face and the mind. New York. Oxford University Press 1999. 37-60.

10. Tarkkila L, Linna M, Tiitinen A, Lindqvist C, Meurman JH. Oral symptoms at menopausethe role of hormone replacement therapy. Oral Surg Oral Med Oral Pathol Oral Radiol Endod 2001; 92: 276-80.

11. Sardella A, Uglietti D, Demarosi F, Lodi G. Enjuagues orales con cloruro de benzidamina en el manejo del síndrome de ardor bucal. Oral Surg Oral Med Oral Pathol Oral Radiol Endod 1999; 88: 683-6.

12. Pajukoski H., Meurman J.H., Halonen P, Sulkava R. Prevalence of subjective dry mouth and burning mouth in hospitalized elderly patients and outpatients in relation to saliva, medication, and systemic diseases. Oral Surg Oral Med Oral Pathol Oral Radiol Endod. 2001; 92: 641-9.

13. Miyamoto SA, Ziccardi V.B. Burning mouth syndrome. Mt Sinai J Med 1998; 65: 343-7.

14. Grinspan D, Fernández Blanco G, Allevato MA, Stengel FM. Burning mouth syndrome. Int $\mathrm{J}$ Dermatol 1995; 34: 483-7. 
15. Ship JA, Grushka M, Llipton JA, Mott AE, Sessle BJ, Dioner RA. Burning Mouth Syndrome: an update. J Am Dent Assoc 1995; 126: 842-53.

16. Cibirka RM, Nelson SK, Lefebvre CA. Burning mouth syndrome: a review of etiologies. J Prosthet Dent 1999; 78: 93-7.

17. Bergdahl M., Bergdahl J. Burning mouth syndrome: prevalence and associated factors. J Oral Pathol Med 1999; 28: 350-4.

18. Sevensson P, Kaaber S. General health factors and denture function in patients with burning mouth syndrome and matched control subjects. J Oral Rehabilit 1995; 22: 887-95.

19. Lamey PJ, Lewis MAO Oral medicine in practice: burning mouth syndrome. Br Dent J 1989; 167: 197-200.

20. Caballero R. Estomatodinia: boca ardiente o quemante. Avances en Odontoestomatología. 1999; 15: 35-40.

21. Rodrigo MA, García JA. Síndrome de boca ardiente. Gaceta Dental 2000; 104: 86-88.

22. http://www.tastelab.org (Escuela Universitaria de Yale: Laboratorio de la medicina del gusto).

23. Frutos R, Rodríguez S, Miralles L, Machuca G. Manifestaciones orales y manejo odontológico durante la menopausia. Medicina Oral 2002; 7: 26-35.

24. Bascones A, Machuca G. Psicalgias y glosodinia. En: Bascones A, Manso FJ. Dolor orofacial: diagnóstico y tratamiento. Madrid. Avances Médicodentales S.L. 1997. 329-42.

25. Okeson JP. Dolor orofacial según Bell. Barcelona. Quintessence, S.L. 1999. 229-34.

26. Somacarrera ML, Pinos H, Hernández G, Lucas M. Síndrome de la boca ardiente. Aspectos clínicos y perfil psicológico asociado. Archivos de Odontoestomatología 1998; 11: 295-306.
27. Peñarrocha $M$, Peñarrocha $M A$. Síndrome de boca ardiente, algia facial atípica y aspectos psicosomáticos del dolor orofacial. En: Peñarrocha Diago M. Dolor orofacial, etiología, diagnóstico y tratamiento. Barcelona. Masson S.A. 1997. 209-16.

28. Samaranayake LP, Chen $Q$. Growth of the fungal pathogen candida in parotid saliva of patients with BMS. Microbios 2002; 2: 45-52

29. Gall-Trosel K, Mravak-Stipetic M, Jurak I, Ragland WL, Pavelic J. Helicobacter pylori colonization of tongue mucosa-increased incidence in atrophic glossitis and burning mouth syndrome. J Oral Pathol Med 2001; 30: 560-3.

30. Silvestre F. El síndrome de boca ardiente en el paciente geriátrico. En: Bullón P, Velasco E. Odontoestomatología Geriátrica. La atención odontoestomatológica integral al paciente de edad avanzada. Madrid. IMEC. 1996.

31. Paterson AJ, Lamb AB, Clifford TJ, Lamey PJ. Burning mouth syndrome: the relationship between the HAD scale and parafunctional habits. J Oral Pathol Med 1995; 24: 289-92.

32. Thomsom WM, Chalmers JM, Spencer AJ, Ketabi $M$. The occurrence of xerostomia and salivary gland hypofunction in a populationbased sample of older South Australians. Spec Care Dentist 1999; 19: 20-3.

33. Narhi TO, Meurman JH, Ainamo A. Xerostomia and hyposalivation: causes, consequences and treatment in the elderly. Drugs Aging 1999; 15: 103-16.

34. Bergdahl M. Salivary flow and oral complaints in adult dental patients. Community Dent Oral Epidemiol 2000; 28: 59-66.

35. Anttila SS, Knuuttila ML, Sakki TK. Depressive symptoms as an underlying factor of the sensation of dry mouth. Psychosom Med 1998; 60: 215-8.

36. Fisher D, Ship JA. Effect of age on variability of 
parotid salivary gland flow rates over time. Age and Ageing 1999; 28: 557-61.

37. Bergdahl J, Bergdahl M. Environmental illness: evaluation of salivary flow, symptoms, diseases, medications, and psychological factors. Acta Odontol Scand 2001; 59: 104-10.

38. Bergdahl M. Salivary flow and oral complaints in adult dental patients. Community Dent Oral Epidemiol 2000; 28: 59-66.

39. Lundy FT, Al-Hashimi I, Rees TD, Lamey PJ. Evaluation of major parotid glycoproteins in patients with burning mouth syndrome. Oral Surg Oral Med Oral Pathol Oral Radiol Endod 1997; 83: 252-8.

40. Dubois-Gusnet $\mathrm{CH}$. The oral mucosa and delayed hypersensitivity. Allerg Inmunol 2000; 34: 19 21.

41. Eisen D, Eisemberg E. Oral lichen planus and burning mouth syndrome. Is there a role for path testing? Am J Contac Dermat 2000; 1: 111-4.

42. Formarker BK, Mott AE, Frank ME. The effects of topical anesthesia on oral burning in burning mouth syndrome. Ann N Y Acad Sci 1998; 30: 776-80.

43. Lamey PJ, Hobson RS, Orchardson R. Perception of stimulus size in patients with burning mouth syndrome. J Oral Pathol Med 1996; 25: 420-3.

44. Ito M, Kurita K, Ito T, Arao M. Pain threshold and pain recovery after experimental stimulation in patients with burning mouth syndrome. Psychiatry Clin Neurosci 2002; 56: 161-8.

45. Gao S, Wang Y, Wang Z. Assessment of trigeminal somatosensory evoked potentials in burning mouth syndrome. Chin J Dent Res 2000; 3: 40-6.

46. Bartoshok LM, Grushka M, Duffy VB, Fast L, Prutkin J. Burning mouth syndrome: damage to $\mathrm{CN}$ VII and pain phantoms in CN V (Abstract). Chem Senses 1999; 24: 609.
47. Formarker BK, Frank ME. Taste function in patients with oral burning. Chem Sciences 2000; 25: 575-81.

48. Vucicevic-Boras V, Topic B, Cekic-Arambasin A, Zadro R, Stavljenic-Rukavina A. Lack of association between burning mouth syndrome and hematinic deficiencies. Eur J Med Res 2001; 28: 409-12.

49. Osaki T, Ueta E, Arisawa K, Kitamura Y, Matsugi $N$. The pathophysiology of glossal pain in patients with iron deficiency and anemia. The American Journal of the medical sciences 1999; 318: 324-9.

50. Carrington J, Getter L, Brown RS. Diabetic neuropathy masquerading as glossodynia. J Am Dent Assoc 2001; 132: 1549-51.

51. Pokupec-Gruden JS, Cekic-Arambasin A, Gruden V. Psychogenic factors in the aetiology of stomatopyrosis. Coll Antropol 2000; 24: 119-26.

52. Bergdahl J, Anneroth G, Perris H. Personality characteristics of patients with resistant BMS. Acta Odontol Scand 1995; 53: 7-11.

53. Grushka M, Epstein J, Mott A. An open-label, dose escalation pilot study of the effect of clonazepam in burning mouth syndrome. Oral Surg Oral Med Oral Pathol Oral Radiol Endod 1998; 86: 557-61.

54. Katzung BG, Trevor AJ. Pharmacology: examination and board review. 4th ed. Norwalk, Conn: Appleton and Lange. 1995; 214-8.

55. Cullhane NS, Holde AD. Burning mouth syndrome after taking clonazepam. Am Pharmacother 2001; 35: 874-6.

56. Eguía del Valle A, Aguirre Urízar jm, Sagasta Pujana O. Tratamiento del Síndrome de Boca ardiente: Análisis crítico de la literatura y propuesta de un protocolo. Revista Vasca de Odontoestomatología 2002; 12: 24-30.

57. Sardella A, Carrassi A. BMS: S for syndrome or S 
for symptom? A reappraisal of the burning mouth syndrome. Minerva Stomatol 2001; 50: 241-6.

58. Chimenos Küstner E, Marques Soares MS. Boca ardiente y saliva. Medicina Oral 2002; 7: 244-53.

\section{CORRESPONDENCIA}

Dr. Eduardo Chimenos Küstner Vía Augusta $124,1^{\circ} 3^{a}$ 08006 - BCN

E-mail: 13598eck@comb.es 